\title{
Antibiotics Friend and Foe: "From Wonder Drug to Causing Mitochondrial Dysfunction, Disrupting Human Microbiome and Promoting Tumorigenesis"
}

\author{
Robert L. Elliott ${ }^{1,2}$, Xian P. Jiang' ${ }^{1}$, Catherine Baucom² ${ }^{2}$, Zuzanna Lomnicka1 \\ ${ }^{1}$ Sallie Astor Burdine Breast Foundation, Baton Rouge, USA \\ ${ }^{2}$ Elliott Mastology Center, Baton Rouge, USA \\ Email: drrobertelliott@cox.net
}

How to cite this paper: Elliott, R.L., Jiang, X.P., Baucom, C. and Lomnicka, Z. (2018) Antibiotics Friend and Foe: "From Wonder Drug to Causing Mitochondrial Dysfunction, Disrupting Human Microbiome and Promoting Tumorigenesis". International Journal of Clinical Medicine, 9, 182-186. https://doi.org/10.4236/ijcm.2018.93016

Received: February 22, 2018

Accepted: March 23, 2018

Published: March 26, 2018

Copyright $\odot 2018$ by authors and Scientific Research Publishing Inc. This work is licensed under the Creative Commons Attribution International License (CC BY 4.0).

http://creativecommons.org/licenses/by/4.0/

\begin{abstract}
Mitochondria are evolutionary bacteria that are dynamic intracellular organelles involved in many vital cellular functions. However, modern medicine has fallen prey to misuse and over-usage of antibiotics. This misuse can damage the mitochondrion, alter host antibiotic interactions, and cause serious pathophysiologic conditions. We believe this leads to mitochondrial dysfunction, which may promote tumorigenesis and neurodegeneration. This opinion commentary's goal is to bring awareness of this important hot topic to the medical community before induced modern plagues are irreversible.
\end{abstract}

\section{Keywords}

Mitochondrial Dysfunction, Microbiome, Tumorigenesis, Endosymbiotic, Warburg Effect

We recently published a paper entitled "Antibiotic Overusage Causes Mitochondrial Dysfunction which May Promote Tumorigenesis" [1]. Some have considered this paper very controversial; however, it has been well received in the cancer metabolism community. We decided to write this commentary after reading a book entitled "Missing Microbes: How the Overusage of Antibiotics is Fueling Our Modern Plagues" written by Dr. Martin J. Blaser [2]. This book is a must for anyone interested in the human microbiome and its role in maintaining a balance of physiologic function and health. However, we knew we needed to write it after attending the American Association for Cancer Research's 
(AACR) "Tumor Immunology and Immunotherapy" meeting in Boston October $1^{\text {st }}-4^{\text {th }} 2017$. That meeting convinced us that the impact of antibiotic overusage on many aspects of human health is being ignored by the medical community. We are presently evaluating the effect of antibiotics on mitochondrial function and possible initiation of tumorigenesis.

Mitochondria are dynamic intracellular organelles involved in many vital cellular functions. Human health depends on good mitochondrial health and biogenesis. Many neurodegenerative diseases, such as Alzheimer's, Parkinson's, and amyotrophic lateral sclerosis, are associated with mitochondrial dysfunction. In the 1930s, Warburg reported that mitochondrial dysfunction was related to tumorigenesis. He discovered that cancer cells had defective respiration and increased glycolysis and lactate production even in the presence of oxygen. This aerobic glycolysis became known as the "Warburg Effect" [3] [4].

Lynn Margulis postulated many years ago that mitochondria are probably descended from free living bacteria that survived endocytosis by a eukaryotic host cell over a billion years ago. This union created a tremendous endosymbiotic relationship [5]. Michael Gray has done great work on mitochondrial evolution and has described DNA and hard scientific evidence affirming a bacterial origin of mitochondria [6]. This fact prompted us to postulate that if they were bacteria they might act like bacteria. Thus, antibiotics could possibly affect and damage mitochondria just as they do bacteria.

Dr. Blaser has done a marvelous and tremendous job describing the importance of the human microbiome in human health. He explains how bacteria are probably the earliest life forms that evolved from the early oceans. There are probably billions of bacterial species. They come in all shapes and are complete cells that have various types of metabolism. Some can live in harsh environments, and are extremely adapted for survival in this world. Many have developed a healthy symbiotic relationship with us humans and when that relationship is disrupted, we are in trouble. He points out that in the past few decades with medical advances something has gone wrong and people are getting sicker. He states "we are suffering from a mysterious array of what he calls modern plagues: obesity, childhood asthma, hay fever, food allergies, esophageal reflux, cancer, celiac disease, Crohn's disease, ulcerative colitis, autism, and eczema." These plagues are chronic and disrupt the quality of life of all of its victims for many years. Dr. Blaser describes how the overuse of antibiotics can contribute to damage of the human microbiome and cause any of these modern plagues. The antibiotics affect our bacterial microbiome directly and Dr. Blaser elegantly explains how and why. However, to the best of our knowledge, he does not address the harm that antibiotics do to the bacteria's ancient evolutionary relative the mitochondrion. This antibiotic damage causes mitochondrial dysfunction and, in our opinion, contributes to tumorigenesis.

Dr. Blaser discusses a magnitude of triumphs, severe problems, and failures caused by antibiotics. He talks about the wonder years when doctors thought an- 
tibiotics were great to eliminate serious infections and many lives were saved. They were used frequently because of no known consequences. This, of course, was very untrue. Resistant bacteria emerged and now present severe problems. Resistant bacteria are increasing and are causing many deaths. Overuse of antibiotics kills and disrupts our good flora in the microbiome, which Blaser states, is another human organ. This problem is magnified by the use of broad spectrum antibiotics, and is complicated because drug companies are not producing enough new antibiotics.

It is not just the overuse in humans, but also how we treat farm animals and contaminate our food supply. This practice is especially dangerous to children, disrupting their developing microbiome. He emphasized the importance of vaginal birth and the importance of the baby being bathed and massaged by the Mother's vaginal bacterial flora. The rise in C-sections has cheated babies out of exposure to these bacteria important in the birth of their extremely important microbiome. He even points out that the gut microbiota plays a significant role during a woman's pregnancy and may even be involved in inducing labor.

Dr. Blaser believes we do have solutions because the antibiotic era began only 70 years ago which is a very short period in the evolution of the human microbiome. He does, however, think we are at the tipping point and action should be taken now or we will enter an "Antibiotics Winter". He states we must decrease our appetite for antibiotics and stop so much the use of hand sanitizers. With sick children, the doctor must reconstruct daily events before the illness. He or she really needs to examine the child, parents need to be patient, and all parties should be better informed. Antibiotics should only be used when necessary. We also need to stop farmers from giving antibiotics to our food supply, develop quicker and better diagnostic techniques, and encourage more and better drug development [2].

Our paper on antibiotic overusage causing mitochondrial dysfunction and possibly promoting tumorigenesis discussed the following topics: 1) Common problems caused by antibiotics, such as antibiotic resistance, 2) Antibiotic effect on host immunity (C-Diff) and vaginitis, 3) Effect on gut microbiota and efficacy of cancer immunotherapy with checkpoint inhibitors, 4) Adverse effects of antibiotics on mitochondria. The latter of which we will present preliminary research evidence to support that antibiotic damage to mitochondria may promote tumorigenesis [1].

There are definitely adverse effects of antibiotics on mitochondria. The fact that mitochondria are archaic bacterial ancestors explains why mitochondrial damage is caused by the drugs antibacterial effects. Many antibiotics are inhibitors of bacterial ribosomes and may also inhibit mitochondrial ribosomes. It is thought that their effects on mitochondria are because of the shared targets of prokaryotes and mitochondria [7]. These side effects can cause defective cellular respiration, mitochondrial dysfunction, and induce the glycolytic pathway. This pathway many promote tumorigenesis. 
We will now present some preliminary laboratory evidence to support this hypothesis. In conventional cell culture, gentamicin is added to culture media to prevent bacterial contamination. We used Real-time PCR (polymerase chain reaction) to compare the gene expression of human normal mammary epithelial MCF-12A cells grown in culture media without gentamicin to cells grown in media with $0.05 \mathrm{mg} / \mathrm{mL}$ gentamicin. We found that gentamicin upregulated mRNA expression of hypoxia-inducible factor 1 alpha (HIF $1 \alpha$ ) 3 fold, glucose transporter 3 (SLC2A3) 19 fold, and glycolytic genes lactate dehydrogenase A (LDHA) 2.1 fold and phosphofructokinase 1 (PFKM) 2.6 fold (Figure 1). This suggest gentamicin induces aerobic glycolysis and increases glucose uptake in human normal mammary epithelia. Many in vitro experiments have been done in cell cultures with gentamicin addition, which suggests that published data may have been obtained in glycolytic cells and results may be skewed.

Our preliminary research data was obtained in gentamicin free cell culture media. Azithromycin is a common antibiotic used to treat human bacterial infections. We tested the effects of azithromycin on gene expression of the MCF-12A cell line. Real time PCR showed that azithromycin significantly increased mRNA expression of HIF1 $\alpha$, glycolytic enzymes and glucose transporters 1 and

\section{MCF-12A}

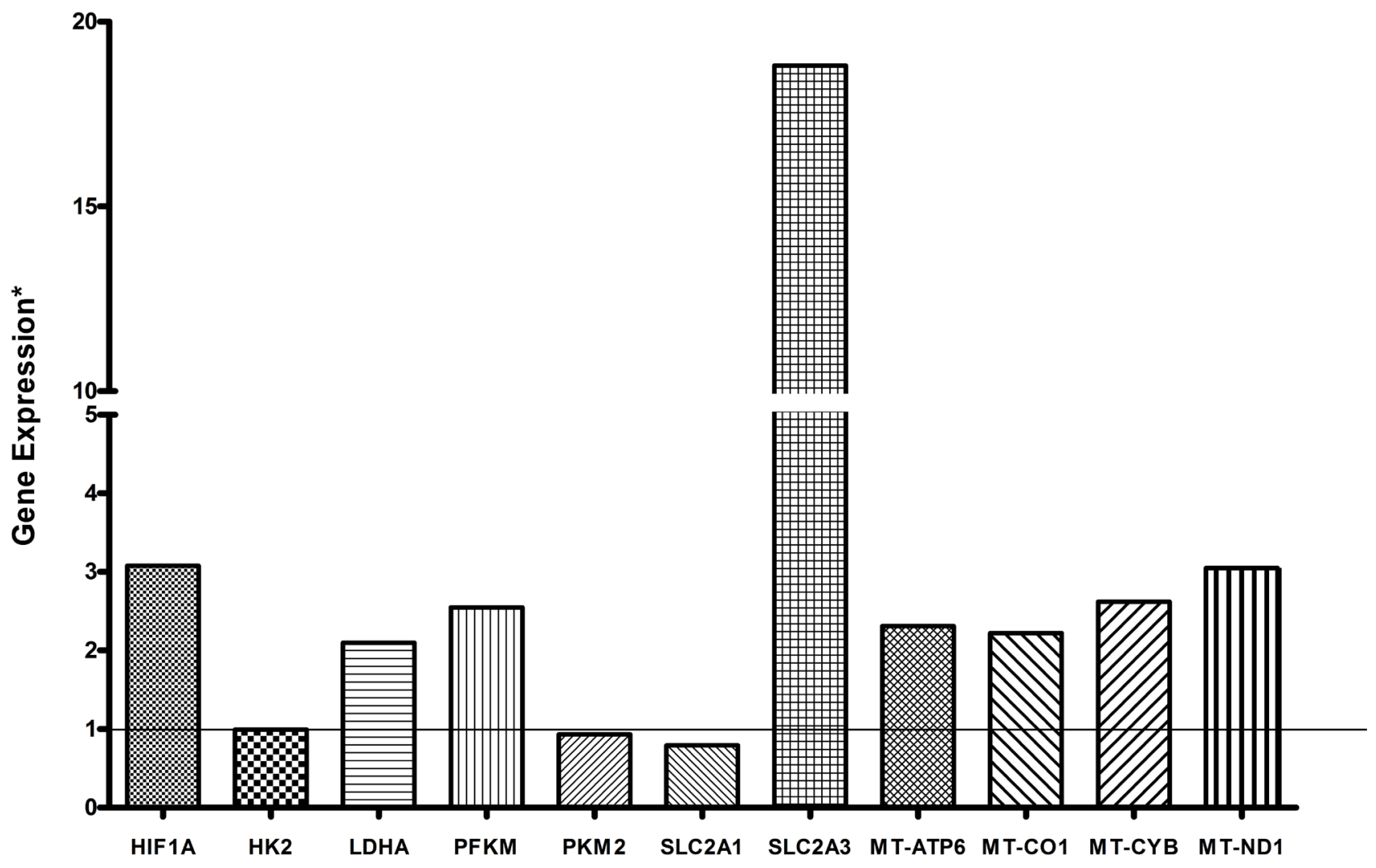

Figure 1. Gentamicin addition in the culture medium changed gene expression of MCF-12A cells. Gene expression (mRNA) was measured by real time PCR. ${ }^{\star}$ Ratio of the cells cultured in medium with gentamicin addition to cells cultured in gentamicin free medium. Ratio $>1$ indicates an increase in gene expression. 
3 after 7 days of incubation. These results confirmed azithromycin can induce aerobic glycolysis in MCF-12A cells (date not shown). We also found that the mRNA expression of mitochondrial oxidative phosphorylation (OXPHOS) enzymes were significantly increased after 7 days (date not shown). This increase in mitochondrial OXPHOS increases reactive oxygen species which can lead to mitochondrial and nuclear DNA instability. Induced aerobic glycolysis and DNA mutation have been shown to contribute to tumorigenesis. Increased reactive oxygen species are also associated with other conditions, such as amyotrophic lateral sclerosis (ALS), Alzheimer's, and age.

In summary, Dr. Blaser has outlined in his book many ways the overuse of antibiotics can disrupt the human microbiome and the serious consequences of that disruption. Several respected great scientists presented papers at the (AACR) meeting in Boston discussing the importance of the human microbiome in cancer and cancer immunotherapy response, especially to checkpoint inhibitors. However, none emphasized how the effect of antibiotic use might impact the microbiome and response to therapy.

Neither Dr. Blaser nor any of the scientists in Boston mentioned any association antibiotics might have on the bacterial relative "the mitochondrion". Our preliminary research confirms that antibiotics can affect mitochondrial glucose transporters and glycolytic and OXPHOS enzymes. Recent live cell imaging of the MCF-12A cell line has shown that azithromycin causes marked increase of nuclear and mitochondrial reactive oxygen species (ROS). This increase in ROS) may cause nuclear and mtDNA instability, and thus promote tumorigenesis (data not shown).

We have more work in progress, but we are nearly convinced that antibiotic abuse not only disrupts the human microbiome, but probably also promotes tumorigenesis. It is time the medical community starts addressing this serious problem. Stay tuned more to come.

\section{References}

[1] Elliott, R.L., Jiang, X.P. and Baucom, C.C. (2017) Antibiotic Overusage Causes Mitochondrial Dysfunction Which May Promote Tumorigenesis. Journal of Cancer Treatment and Research, 5, 62-65. https://doi.org/10.11648/j.jctr.20170504.11

[2] Blaser, M.J. (2014) Missing Microbes: (How the Overusage of Antibiotics Is Fueling Our Modern Plagues) Henry Holt.

[3] Warburg, O., Wind, F. and Negleis, E. (1930) On the Metabolism of Tumors in the Body. In: Warburg, Ed., The Metabolism of Tumors Constable, Princeton, 254-270.

[4] Warburg, O. (1956) On the Origin of Cancer Cells. Science, 123, 309-314. https://doi.org/10.1126/science.123.3191.309

[5] Margulis, L. (1970) Origin of Eukaryotic Cells. Yale University Press, New Haven.

[6] Gray, M.W. (2012) Mitochondrial Evolution. Cold Spring Harbor Perspectives in Biology, 4, 1403. https://doi.org/10.1101/cshperspect.a011403

[7] Barnhill, A.E., Brewer, M.T. and Carlson, S.A. (2012) Adverse Effects of Antimicrobials via Predictable or Idiosyncratic Inhibition of Host Mitochondrial Components. Antimicrobial Agents and Chemotherapy, 56, 4046-4051. https://doi.org/10.1128/AAC.00678-12 